\title{
Novel approach for hybrid MAC scheme for balanced energy and transmission in sensor devices
}

\author{
Anitha Krishna Gowda ${ }^{1}$, Ananda Babu Jayachandra ${ }^{2}$, Raviprakash Madenur Lingaraju ${ }^{3}$, \\ Vinay Doddametikurke Rajkumar ${ }^{4}$ \\ ${ }^{1}$ Department of Computer Science and Engineering, Global Academy of Technology, Bengaluru, India \\ ${ }^{2}$ Department of Information Science and Engineering, Malnad College of Engineering, Hassan, India \\ ${ }^{3}$ Department of Computer Science and Engineering, Kalpataru Institute of Technology, Tiptur, India \\ ${ }^{4}$ Department of Computer Science and Engineering, Channabasaveshwara Institute of Technology, Gubbi, India
}

\begin{abstract}
Article Info
Article history:

Received Oct 29, 2020

Revised Jul 3, 2021

Accepted Jul 18, 2021

Keywords:
Collision
Energy saving
Hybrid MAC protocol
Network lifetime
Wireless sensor network

Keywords:

Collision

Energy saving

Network lifetime

Wireless sensor network

ABSTRACT

Hybrid medium access control (MAC) scheme is one of the prominent mechanisms to offer energy efficiency in wireless sensor network where the potential features for both contention-based and schedule-based approaches are mechanized. However, the review of existing hybrid MAC scheme shows many loopholes where mainly it is observed that there is too much inclusion of time-slotting or else there is an inclusion of sophisticated mechanism not meant for offering flexibility to sensor node towards extending its services for upcoming applications of it. Therefore, this manuscript introduces a novel hybrid MAC scheme which is meant for offering cost effective and simplified scheduling operation in order to balance the performance of energy efficiency along with data aggregation performance. The simulated outcome of the study shows that proposed system offers better energy consumption, better throughput, reduced memory consumption, and faster processing in contrast to existing hybrid MAC protocols.
\end{abstract}

This is an open access article under the CC BY-SA license.

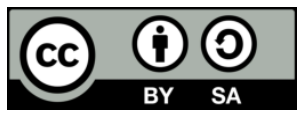

\section{Corresponding Author:}

Anitha Krishna Gowda

Department of Computer Science and Engineering, Global Academy of Technology

Bengaluru, India

Email: anitharesearchvtu@gmail.com

\section{INTRODUCTION}

Wireless sensor network is known for its cost effective mechanism for extracting information from the surroundings in form of physical attributes [1]. The extracted information is forwarded to the user via the process of data fusion and data aggregation after a clustering is carried out [2]. In this entire process of capturing the data to forwarding the data, significant amount of energy is drained as well as still there are extent of data collision in the large and distributed environment of deployment. At present, there are various studies associated with data quality [3] and energy efficiency [4] where various forms of approaches have been presented till date. Out of all the approaches, the medium access control (MAC) scheme is witnessed to offer more capability to deal with this situation. The prime responsibility of MAC protocol is to make a decision for a favorable condition for accessing the shared medium for resisting possibility of collision while perform data aggregation [5]. This is very much essential as a sensor node doesn't have higher degree of processing capability nor it has any extensive resource availability, hence, a better form of performance can be only ensured if they operate by accessing sharing medium. The fact is when the sensor nodes is in idle condition, there is higher consumption of resources compared to active state of communication. The operational features of the radio of sensor node are directly controlled by MAC protocol and it formulates a 
decision for accessing the shared medium. When a sensor node executes MAC protocol, it witnesses idle listening, over heading, overhead of protocol, and fluctuation of traffic that causes drainage of energy. It will also mean that there will be extensive drainage of energy by the medium access and therefore, it is essential to consider energy efficient as core objective while working on MAC protocol design. As per theory and deployment, there are various forms of MAC protocols [6] viz. i) contention based and ii) schedule based. In contention-based MAC scheme, there is no dependency towards central coordination of sensors in order to access medium. Some of the examples are sensor MAC (S-MAC) [7], Berkley MAC for Low power sensor network (B-MAC) [8], predictive wake up MAC (PW-MAC) [9]. In schedule-based MAC, the medium access is arbitrated using an explicit order or schedule in order to facilitate transmission. Some of the examples of schedule-based MAC are low-energy adaptive clustering hierarchy (LEACH) [10], Powerefficient and delay aware medium access protocol [11], priority-based MAC (PRIMA) [12]. However, both this MAC protocols have been witnessed with advantageous features as well as challenges which are overcomed in hybrid MAC protocols which uses both the schemes in order to offer access to shared medium. Hybrid MAC protocols are of two types of mainly zebra MAC (Z-MAC) [13] and advertisement MAC (AMAC) [14]. However, hybrid MAC scheme is comparatively new and there is less investigation carried out in order to evaluate their applicability over various test environments with challenging scenario. Such protocols are in beginning level of deployment in the advanced application of wireless sensor network where more work is required to facilitate smarter slot management and effective scheduling practices. Therefore, the proposed system offers a discussion a novel hybrid MAC system which unlike any existing system offers better scheduling scheme. The prime agenda of this implementation is also to evolve up with an integrated framework with a special emphasis towards balance between energy conservation as well as data aggregation performance. Section 1 discusses about the existing literatures where different techniques of hybrid mac scheme over various test environment are discussed for detection schemes used in data aggregation for sensory application followed by discussion of research problems and proposed solution. Section 2 discusses about algorithm implementation where primary and secondary hybrid MAC scheme is discussed and elaborated followed by discussion of result analysis with more detailing about comparison in section 3 . Finally, the conclusive remarks are provided in section 4.

At present, there are various hybrid MAC approaches used in wireless sensor network and are discussed in our work [15]. The recent work carried out by Al-Janabi et al. [16] have discussed about a stochastic model for hybrid MAC using Markov a model where the energy performance is improved significantly. Ruan et al. [17] have performed a comparative study for different forms of network in order to assess the efficiency of hybrid MAC protocol. Existing studies has also seen various case studies where hybrid MAC approach has been used. A unique study carried out by Madi and Al-Qamzi [18] have presented a MAC approach for hybrid network system considering mobility factor while work of Zhang et al. [19] have considered for underwater sensory application where the slot management is carried out on the basis of traffic load. The discussion presented by Saboor et al. [20] have disclosed certain open end research issues associated with used of hybrid MAC in special cases of application. Ammar et al. [21] have presented a cooperative scheme with single relay in distributed manner with a sole purpose of minimizing the energy consumption and assured quality of service. Haqbeen et al. [22] have used the dynamic scheduling for resisting delay mainly while applying hybrid MAC approach. Study towards optimization of network performance has been studied by $\mathrm{Hu}$ et al. [23] where a different form of wireless network has been studied to find that it offers maximum capacity. A case study of industrial application has been taken by Meinyk et al. [24] where the study found different hybrid MAC applicable over resource constrained wireless transmission. Saad et al. [25] have offered an optimized scheme focusing on machine-to-machine communication using hybrid MAC system where Monte-Carlo simulation has been used. Yang et al. [26] have integrated conventional time division multiple access (TDMA) and carrier sense multiple access with (CSMA) scheme addressing energy issue and throughput issue in mobility scenario of sensory application. Deng et al. [27] have used contention-based MAC approach on lightweight network and reservation-based approach on heavy network. Ma et al. [28] have presented a unique scheduling practice that uses physical parameters of sensory application to find that it offers better data delivery. Another unique MAC approach has been introduced by Jeon et al. [29] for optimizing the memory system which can be applicable on any architecture associated with data streams. Cao et al. [30] has carried out investigation towards reservation system of multiple numbers of slots. The work of Alam and Kim [31] has emphasized on heterogeneous sensor network and developed a hybrid MAC protocol that makes use of directional antenna. Nguyen et al. [32] have developed a hybrid MAC approach meant for eliminating surplus beacons to reduce overhead. Apart from this, there are various other approaches e.g., Yang et al. [33], Liu et al. [34], Hu et al. [35], Hamiane et al. [36]. Therefore, it can be seen that there has been extensive work carried out towards hybrid MAC approach considering different use cases where majority of the work has focused on energy problems in wireless network. The above discussed work has its advantages claimed in the papers itself; however, its 
identified issues after reviewing are briefed in next section. The significant research problems are as follows: i) existing approaches has not assessed the applicability of hybrid MAC over the variable traffic load scenario which renders them unapplicable over real-time traffic system; ii) inclusion of more involvement of slots in developing hybrid MAC scheme incurs extensive resource usage which degrades the communication performance over a long run; iii) although scheduling is carried out in existing system but they are not found to offer supportability of collision avoidance and interference; iv) there are less number of hybrid MAC scheme which balances energy efficiency along with data transmission performance in wireless sensor network.

Therefore, the problem statement of the proposed study can be stated as "developing a simplified hybrid MAC scheme with a unique scheduling scheme to offer a better form of equilibrium between data aggregation performance and energy efficiency". With continuation of our prior work [37], [38] the main goal of the proposed system is to design and develop a novel hybrid MAC approach that could offer an optimal performance for data aggregation in wireless sensor network. Figure 1 highlights the implementation scheme of proposed system where it can be seen that it initiates from a neighborhood discovery of the sensor nodes followed by a unique and simplified clustering operation unlike any existing system. This operation is further followed by a synchronization process which is about managing an effective time slot suitable in dynamic order. This operation is essentially meant for resisting an event of collision while performing routing operation. The next part of the planning of proposed design consists of selection of intermediate sensors where the preference is given for the nodes occur in single hop which is considered information about location, energy, and buffer. A simplified energy modeling is design, which ensures to restrict and fine tune the transmittance energy as per the demand of the current data forwarding. Finally, a logical condition is formulated which ensures that selection process of an intermediate nodes.

An analytical methodology is adopted in order to construct the proposed system. It should be noted that proposed system mainly aims for energy efficiency by ensuring that complete mechanism of the data aggregation process obeys the principle of a novel scheduling process. Another significance of the proposed system is also to offer a good performance of the data delivery along with the energy efficiency in presence of any challenging traffic load situation in wireless sensor network. The next section discusses about the algorithm description.

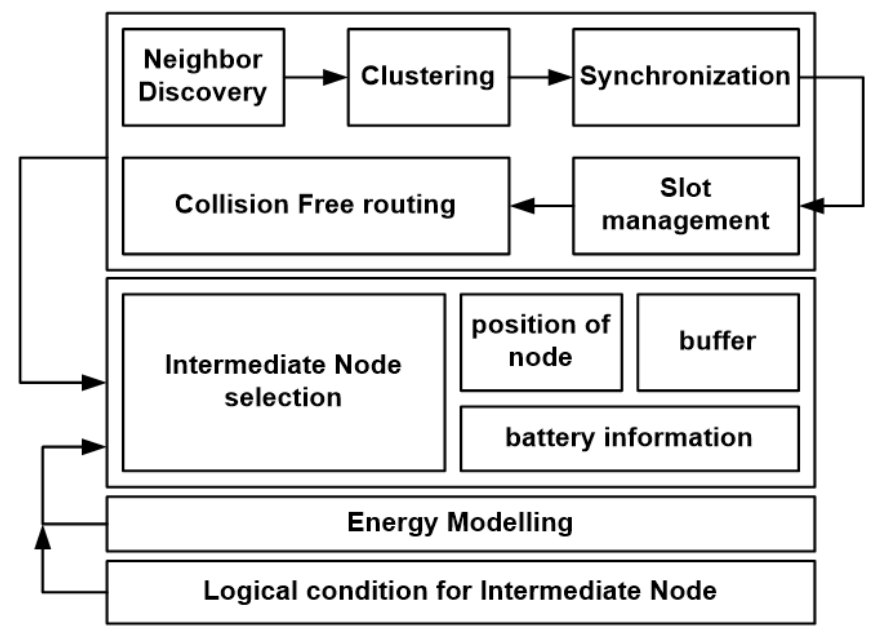

Figure 1. Schema of proposed methodology

\section{ALGORITHM IMPLEMENTATION}

The complete implementation of the proposed system is carried out in two stages of implementation viz. primary and secondary hybrid MAC scheme. The complete focus of the algorithm implementation is to offer a good balance between the communication performances along with energy control while performing data aggregation in wireless sensor network. The descriptions of the algorithms are as follows:

\subsection{Algorithm for primary hybrid MAC scheme}

The core responsibility of this primary scheme is to offer a better sleep and awake management for the sensor node to carry out enhanced hybrid MAC scheduling. The algorithm takes the input of number of nodes $n$ in presence of a single base station which can be placed at any position over the simulation area. 
Unlike any clustering approaches in existing system, the algorithm performs clustering on the basis of user input of number of clusters $\mathrm{n}_{\mathrm{c}}$. The operation leads to a yield of data to be transmitted data and energy e. The steps of the algorithms are as follows:

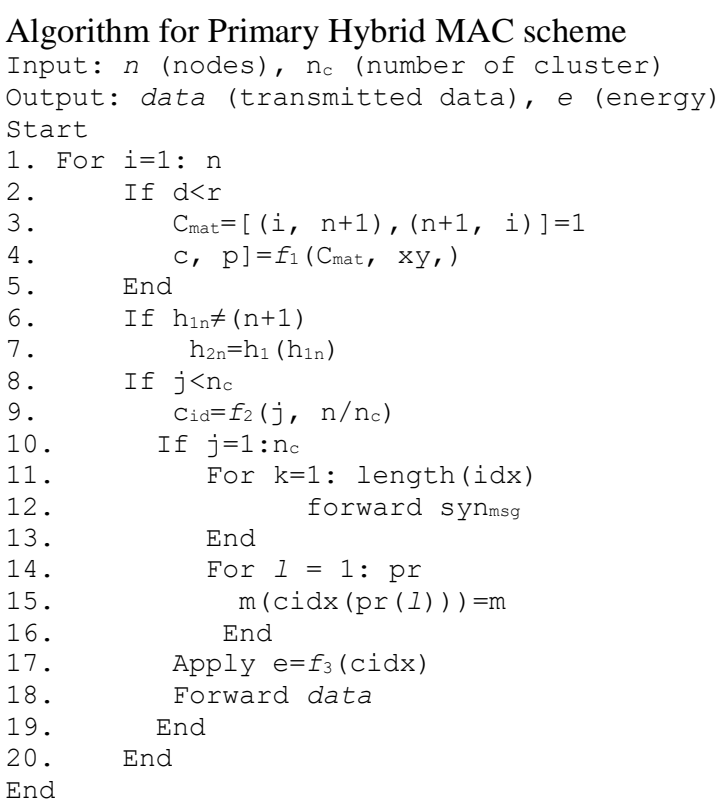

The algorithm takes the $\mathrm{n}$ number of node and dispersed randomly in simulation area. The algorithm computes the distance $d$ among each node (Line-2). A condition is formulated which checks if the nodes come within the transmission range $r$ for each other using Euclidean distance (Line-2). This operation is followed by upgrading the $C_{\text {mat }}$ matrix to unity values. The $C_{\text {mat }}$ matrix stores all the nodes which are within the transmission range of each other (Line-3) as well as it also computes the distance xy between all nodes in its new position with the base station. This operation is followed by applying an explicit function $\mathrm{f}_{1}(\mathrm{x})$ which act as new transmission scheme considering input arguments of matrix $C_{\text {mat }}$ and distance $x y$ (Line-4). This function formulates a communication vector on the basis of shortest path between two nodes with an outcome of cost $\mathrm{c}$ and path $\mathrm{p}$ (Line-4). The next part of the algorithm is to search for double hops $\mathrm{h}_{2 \mathrm{n}}$ from the single hop $\mathrm{h}_{1 \mathrm{n}}$ considering a matrix $\mathrm{h}_{1}$ which has all information of single hop nodes (Line-6 and Line-7). This process is followed by assigning a cluster identity where a function $\mathrm{f}_{2}(\mathrm{x})$ is formed which assigns the random numbers to the communicating nodes considering number of nodes $\mathrm{n}$ and number of clusters (Line9). The consecutive process is to consider all the clusters $n_{c}$ (Line-10) and it extracts all the identity of the nodes which are the part of clusters and it considers the length of that identity (Line-11) followed by forwarding of the sync message synmsg (Line-12). The proposed system also considers different levels of priority and selects the node for participating in data forwarding process on the basis of pr priority level (Line-15). Another function $\mathrm{f}_{3}(\mathrm{x})$ is formulated for energy control for all the nodes inside cluster cidx (Line-17).

\subsection{Algorithm for secondary hybrid MAC scheme}

The primary algorithm discussed mainly about the slot management thereby revving a new form of hybrid MAC protocol considering hop wise communication; however, it is essential to ensure the participation of an intermediate sensor in order to ensure prevention of collision in proposed system. The hybrid nature of the MAC protocol in prior algorithm is further required to ensure extensive participation of all the sensors where intermediate sensors are very much important in the data forwarding process. Therefore, this algorithm is responsible for performing further optimization towards the primary hybrid MAC protocol with respect to energy efficiency. The core idea is to enhance the scalability too while performing communication. The algorithm essentially chooses an intermediate sensor in order to ensure that their communication successfully takes place. The algorithm takes an input of nodes and data to be forwarded which after processing yields $\mathrm{nr}$ finally selected intermediate node. The steps of the algorithm are as follows:

Algorithm for Secondary Hybrid MAC Scheme

Input: $n$ (nodes), data (data to be forwarded)

Output: nr (final relay sensor) 


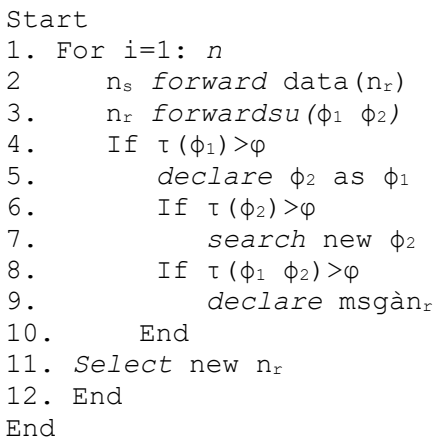

The execution of the proposed algorithm considers similar number of sensors that offers an outcome of number of intermediate nodes $\mathrm{n}_{\mathrm{r}}$ in order to facilitate a robust transmission of the data packet among the sensor node. The proposed system performs an essential consideration that every single hop sensor possesses the discrete information about the intermediate sensors that comes under the proximity of another sensor with single hop. For this purpose, two definitive roles are given to a sensor i.e., a main intermediate node $\phi_{1}$ and support intermediate sensor $\phi_{2}$. The main intermediate sensor is responsible for forwarding as well as obtaining the data packets to and from the intermediate sensors. The intermediate sensor node should be under single hop connection. Considering all the sensors (Line-1), the proposed system entails the transmitting node $n_{s}$ to transmit the data packet to its proximity intermediate node $n_{r}$ (Line-2). Essential information e.g., energy, memory, and location of the sensors are accessed by an intermediate sensor in the form of update information that are connected with both support and main intermediate sensors (Line-3). A simplified logic of movement is considered in proposed system where the distance between two nodes is computed and if that distance is found to be more than a certain limit than the system consider that the node has moved from old to new position within the simulation area (Line-4). It will eventually mean that if the difference in the distance between the main intermediate sensors is greater than cut-off value of $\varphi$ (Line-4) than the algorithm declares the support intermediate node $\mathrm{n}_{\mathrm{s}}$ as main intermediate node $\mathrm{n}_{\mathrm{r}}($ Line-5). However, the new support intermediate sensor $\mathrm{n}_{\mathrm{s}}$ is selected if the difference between the distances in support intermediate sensor is found to be more than cut-off $\varphi$ (Line-6 and Line-7). On the other hand, if the main intermediate node $\mathrm{nr}$ and support intermediate node $\mathrm{ns}$ to exhibit maximized mobility degree in contrast to the cut-off value (Line-8) than the system declares a notification to a main intermediate node (Line-9). This is one of the explicit characteristics of the sensor that is utilized for the purpose of fine-tuning the mobility aspect of the sensors in any form of application. In the complete process, a discrete time slot system is maintained and managed for better optimization.

Therefore, the primary hybrid MAC algorithm introduces a novel scheduling practice in wireless sensor network when they are exposed to a dynamic traffic condition while the secondary hybrid MAC algorithm incorporates more communication efficiency in the form of reliability in data transmission. This is carried out by ensuring that data once forwarded will always reach its destination irrespective of any traffic challenges and in energy efficient manner. The next section discusses about the simulated outcomes obtained from the proposed implementation.

\section{RESULT ANALYSIS}

This section briefs of the simulated outcome of the proposed system that has been scripted in MATLAB. The simulation environment is as follows: The simulation consists of 500 sensors dispersed randomly over $1100 \times 1100 \mathrm{~m}^{2}$ considering initial time for message interval as 1 seconds which is progressively increased to 10 seconds for assessment. As the proposed system is an essentially MAC approach therefore, it is compared with the existing hybrid MAC protocols e.g., Z-MAC [13] and A-MAC [14] where the performance parameters selected are remaining energy, delay, energy consumption, and algorithm processing time.

Figure 2 highlights that proposed system offers better energy efficiency in contrast to existing hybrid MAC protocols. The prime reason behind this is that proposed system emphasizes on energy saving as well as it offers data transmission on the basis of the dynamic slot management which ensure optimal sleep time of the node which is missing in existing hybrid MAC protocols. Similarly, Figure 3 highlights that proposed system offers better throughput too which can be justified by availability of maximum number of sensors as it works on dynamic scheduling process. Moreover, the secondary hybrid MAC approach offers the better participation of the intermediate nodes which ensures better throughput compared to existing system. 
Figures 4 and 5 show that proposed system offers highly reduced energy consumption and processing time as compared to frequently used standard hybrid MAC protocols respectively. The prime reasons behind lower memory consumptions are: i) only optimal number of nodes assists in data forwarding process as per secondary hybrid MAC approach which results in lesser involvement of saturated nodes and ii) the complete operation is based on run time and no intermediate information is stored. However, it is not the case with existing protocols where information about slot management is stored in every node and there is lack of frequent updating operation resulting in saturation state leading to higher memory consumption.

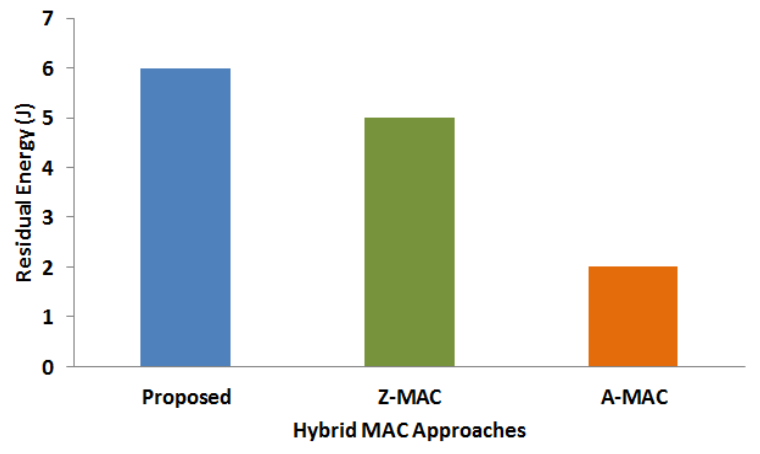

Figure 2. Comparative analysis of residual energy

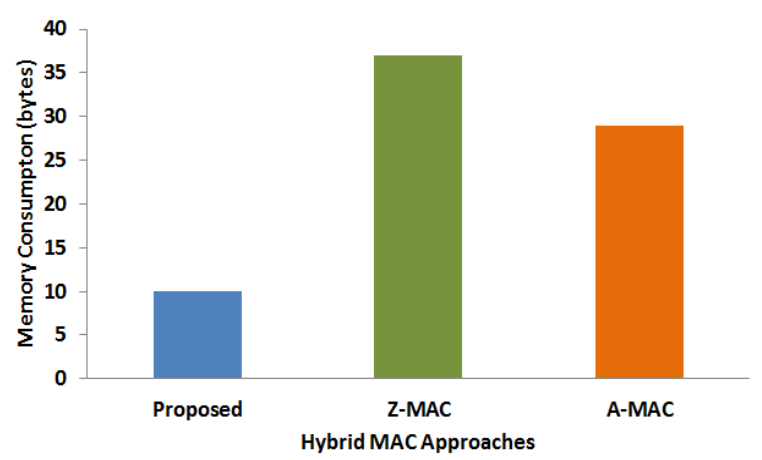

Figure 4. Comparative analysis of memory consumption

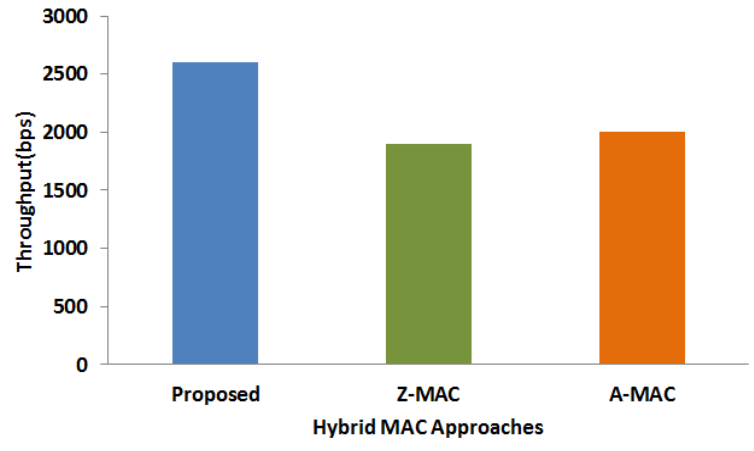

Figure 3. Comparative analysis of throughput

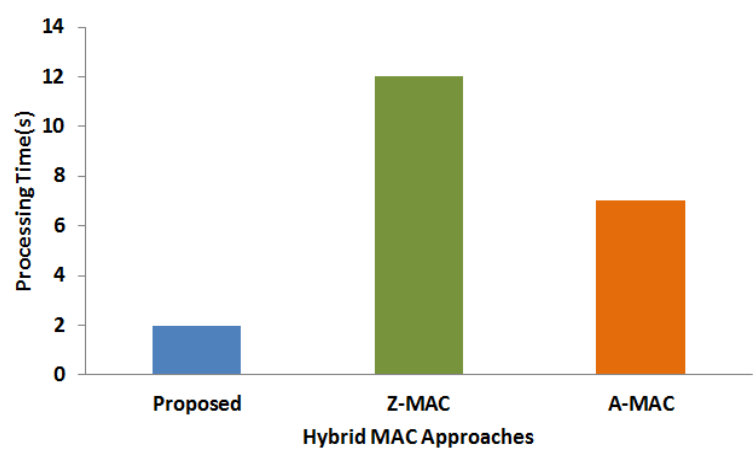

Figure 5. Comparative analysis of processing time

Similarly, processing time of proposed system is very much less due to similar reason. The proposed system offers no iterative operation in forming up communication vector, neither has it offered a typical clustering system like existing clustering approaches with lot of criteria. This saves abundant time in order to formulate network and carry out data aggregation. Hence, the complete mechanism of route discovery as well as management becomes much easier and faster. Owing to this result, it can be said that proposed system could offer supportability to sensory application which is required to be operated in dynamic conditions as it can sustain for longer duration without affecting any data transmission performance. As it offers faster processing duration, it can be used for any time-bound operation with wide ranges of application running over its variable traffic condition.

\section{CONCLUSION}

This paper has presented a unique approach towards constructing an approach where a hybrid MAC scheme is introduced for improving the scheduling scheme which is decentralized in order to accomplish better service quality. The essential contributions of the study are: i) it offers an efficiency in outcome with respect to both passive and active communication in sensor network, ii) it is independent of inclusion of any sophisticated parameters as all the parameters used in modeling are readily available from hop information, iii) the proposed system also uses a simplified and smart slot management as well as user-defined clustering unlike any existing system which is highly flexible as well as customizable. 


\section{REFERENCES}

[1] A. Kumar, M. Zhao, K. Wong, Y. L. Guan and P. H. J. Chong, "A comprehensive study of IoT and WSN MAC protocols: research issues, challenges and opportunities," in IEEE Access, vol. 6, pp. 76228-76262, 2018, doi: 10.1109/ACCESS.2018.2883391.

[2] L. Xu, R. Collier and G. M. P. O'Hare, "A survey of clustering techniques in WSNs and consideration of the challenges of applying such to 5G IoT scenarios," in IEEE Internet of Things Journal, vol. 4, no. 5, pp. 1229-1249, Oct. 2017, doi: 10.1109/JIOT.2017.2726014.

[3] I. Al-Anbagi, M. E. Kantarci and H. T. Mouftah, "A survey on cross-layer quality-of-service approaches in WSNs for delay and reliability-aware applications," in IEEE Communications Surveys \& Tutorials, vol. 18, no. 1, pp. 525-552, Firstquarter 2016, doi: 10.1109/COMST.2014.2363950.

[4] V. L. Quintero, C. Estevez, M. E. Orchard and A. Pérez, "Improvements of energy-efficient techniques in WSNs: A MAC-protocol approach," in IEEE Communications Surveys and Tutorials, vol. 21, no. 2, pp. 1188-1208, Second quarter 2019, doi: 10.1109/COMST.2018.2875810.

[5] M. Khanafer, M. Guennoun and H. T. Mouftah, "A survey of beacon-enabled IEEE 802.15.4 MAC protocols in wireless sensor networks," in IEEE Communications Surveys and Tutorials, vol. 16, no. 2, pp. 856-876, Second Quarter 2014, doi: 10.1109/SURV.2013.112613.00094

[6] T. Kaur and D. Kumar, "QoS mechanisms for MAC protocols in wireless sensor networks: a survey," in IET Communications, vol. 13, no. 14, pp. 2045-2062, 278 2019, doi: 10.1049/iet-com.2018.5110.

[7] W. Ye, J. Heidemann and D. Estrin, "Medium access control with coordinated adaptive sleeping for wireless sensor networks," in IEEE/ACM Transactions on Networking, vol. 12, no. 3, pp. 493-506, Jun. 2004, doi: 10.1109/TNET.2004.828953.

[8] J. Polastre, J. Hill, and D. Culler, "Versatile low power media access for wireless sensor networks," in Proceedings of the Second International Conference on Embedded Networked Sensor Systems (SenSys '04), pp. 95-107, ACMPress, Nov. 2004

[9] L. Tang, Y. Sun, O. Gurewitz and D. B. Johnson, "PW-MAC: An energy-efficient predictive-wakeup MAC protocol for wireless sensor networks," 2011 Proceedings IEEE INFOCOM, 2011, pp. 1305-1313, doi: 10.1109/INFCOM.2011.5934913.

[10] W. B. Heinzelman, A. P. Chandrakasan and H. Balakrishnan, "An application-specific protocol architecture for wireless microsensor networks," in IEEE Transactions on Wireless Communications, vol. 1, no. 4, pp. 660-670, Oct. 2002, doi: 10.1109/TWC.2002.804190.

[11] S. C. Ergen and P. Varaiya, "PEDAMACS: power efficient and delay aware medium access protocol for sensor networks," in IEEE Transactions on Mobile Computing, vol. 5, no. 7, pp. 920-930, Jul. 2006, doi: 10.1109/TMC.2006.100.

[12] J. Ben-Othman, L. Mokdad and B. Yahya, "An energy efficient priority-based QoS MAC protocol for wireless sensor networks," 2011 IEEE International Conference on Communications (ICC), 2011, pp. 1-6, doi: 10.1109/icc.2011.5962414.

[13] I. Rhee, A. Warrier, M. Aia, J. Min and M. L. Sichitiu, "Z-MAC: a hybrid MAC for wireless sensor networks," in IEEE/ACM Transactions on Networking, vol. 16, no. 3, pp. 511-524, Jun. 2008, doi: 10.1109/TNET.2007.900704

[14] S. S. Ray, I. Demirkol and W. Heinzelman, "ADV-MAC: advertisement-based MAC protocol for wireless sensor networks," 2009 Fifth International Conference on Mobile Ad-hoc and Sensor Networks, 2009, pp. 265-272, doi: 10.1109/MSN.2009.27.

[15] K. Anitha and S. Usha, "Research pathway towards MAC protocol in enhancing network performance in wireless sensor network (WSN)," International Journal of Advanced Computer Science and Applications, vol. 8, no. 7, pp. 37-44, 2017.

[16] T. A. Al-Janabi and H. S. Al-Raweshidy, "An energy efficient hybrid MAC protocol with dynamic sleep-based scheduling for high density IoT networks," in IEEE Internet of Things Journal, vol. 6, no. 2, pp. 2273-2287, Apr. 2019, doi: 10.1109/JIOT.2019.2905952.

[17] L. Ruan, M. P. Imali Dias and E. Wong, "Towards low-delay body area networks: an investigation on the hybrid MAC of SmartBAN and IEEE 802.15.6 wireless body area network," 2019 IEEE 20th International Conference on High Performance Switching and Routing (HPSR), 2019, pp. 1-6, doi: 10.1109/HPSR.2019.8808130.

[18] S. Madi and H. Al-Qamzi, "A TDMA-based MAC protocol for hybrid vehicular sensor networks using practical mobility models with real maps," 2019 International Conference on Advanced Electrical Engineering (ICAEE), 2019, pp. 1-6, doi: 10.1109/ICAEE47123.2019.9015104.

[19] Z. Zhang, W. Shi, Q. Niu, Y. Guo, J. Wang and H. Luo, "A load-based hybrid MAC protocol for underwater wireless sensor networks," in IEEE Access, vol. 7, pp. 104542-104552, 2019, doi: 10.1109/ACCESS.2019.2926158.

[20] A. Saboor, R. Ahmad, W. Ahmed, A. K. Kiani, Y. L. Moullec and M. M. Alam, "On research challenges in hybrid medium-access control protocols for IEEE 802.15.6 WBANs," in IEEE Sensors Journal, vol. 19, no. 19, pp. 8543-8555, Oct. 2019, doi: 10.1109/JSEN.2018.2883786.

[21] A. B. Ammar, A. Dziri, M. Terre and H. Youssef, "Hybrid AF/DF based MAC protocol over shadowed channels for wireless sensor networks," 2018 26th International Conference on Software, Telecommunications and Computer Networks (SoftCOM), 2018, pp. 1-5, doi: 10.23919/SOFTCOM.2018.8555821

[22] J. A. Haqbeen, T. Ito, M. Arifuzzaman and T. Otsuka, "Traffic adaptive hybrid MAC with QoS driven energy efficiency for WSNs through joint dynamic scheduling mode," 2018 IEEE/ACIS 17th International Conference on Computer and Information Science (ICIS), 2018, pp. 3-9, doi: 10.1109/ICIS.2018.8466423.

[23] J. Hu, B. Di, Y. Liao, K. Bian and L. Song, "Hybrid mac protocol design and optimization for full duplex Wi-Fi networks," in IEEE Transactions on Wireless Communications, vol. 17, no. 6, pp. 3615-3630, Jun. 2018, doi: 10.1109/TWC.2018.2810119.

[24] S. Melnyk, K. Alam, A. G. Tesfay and H. D. Schotten, "Hybrid MAC for low latency wireless communication enabling industrial HMI applications," 2018 IEEE 4th International Symposium on Wireless Systems within the International Conferences on Intelligent Data Acquisition and Advanced Computing Systems (IDAACS-SWS), 2018, pp. 21-24, doi: 10.1109/IDAACSSWS.2018.8525503.

[25] W. Saad, S. A. El-Feshawy, M. Shokair, and M. I. Dessouky, "Optimised approach based on hybrid MAC protocol for M2M networks," IET Networks, vol. 7, no. 6, 393-397, 2018, doi: 10.1049/iet-net.2017.0240.

[26] X. Yang, L. Wang, J. Su and Y. Gong, "Hybrid MAC protocol design for mobile wireless sensors networks," in IEEE Sensors Letters, vol. 2, no. 2, pp. 1-4, Jun. 2018, Art no. 7500604, doi: 10.1109/LSENS.2018.2828339.

[27] M. Deng, H. Chen and L. Xie, "A hybrid MAC protocol in data-collection-oriented underwater acoustic sensor networks," OCEANS 2017 - Aberdeen, 2017, pp. 1-7, doi: 10.1109/OCEANSE.2017.8084852.

[28] X. Ma, R. Kacimi and R. Dhaou, "Adaptive hybrid MAC protocols for UAV-assisted mobile sensor networks," 2018 15th IEEE Annual Consumer Communications \& Networking Conference (CCNC), 2018, pp. 1-4, doi: 10.1109/CCNC.2018.8319268.

[29] D. Jeon, K. Park and K. Chung, "HMC-MAC: processing-in memory architecture for multiply-accumulate operations with hybrid memory cube," in IEEE Computer Architecture Letters, vol. 17, no. 1, pp. 5-8, Jan.-Jun. 2018, doi: 10.1109/LCA.2017.2700298.

[30] X. Cao, B. Yang, and Z. Song, "Performance analysis of hybrid MAC scheme with multi-slot reservation," Electronics Letters, vol. 54, no. 4, pp. 250-252, 2017, doi: 10.1049/el.2017.2455 
[31] N. Alam and Y.-C. Kim, "Efficient MAC protocol for hybrid wireless network with heterogeneous sensor nodes," Journal of Sensors, vol. 2016, Art. no. 7951965, doi: 10.1155/2016/7951965.

[32] V. Nguyen, T. Z. Oo, P. Chuan and C. S. Hong, "An efficient time slot acquisition on the hybrid TDMA/CSMA multichannel MAC in VANETs," in IEEE Communications Letters, vol. 20, no. 5, pp. 970-973, May 2016, doi: 10.1109/LCOMM.2016.2536672.

[33] M. Yang, M. Gao, C. H. Foh and J. Cai, "Hybrid collision-free medium access (HCFMA) protocol for underwater acoustic networks: design and performance evaluation," in IEEE Journal of Oceanic Engineering, vol. 40, no. 2, pp. 292-302, Apr. 2015, doi: 10.1109/JOE.2014.2311911.

[34] Y. Liu, C. Yuen, X. Cao, N. U. Hassan and J. Chen, "Design of a scalable hybrid MAC protocol for heterogeneous M2M networks," in IEEE Internet of Things Journal, vol. 1, no. 1, pp. 99-111, Feb. 2014, doi: 10.1109/JIOT.2014.2310425.

[35] W. Hu, H. Yousefi'zadeh and X. Li, "Load adaptive MAC: a hybrid MAC protocol for MIMO SDR MANETs," in IEEE Transactions on Wireless Communications, vol. 10, no. 11, pp. 3924-3933, Nov. 2011, doi: 10.1109/TWC.2011.091411.110297.

[36] M. Hamiane and M. J. Ahmed, "Improvement of crankshaft MAC protocol for wireless sensor networks: a simulation study," International Journal of Electrical and Computer Engineering (IJECE), vol. 9, no. 3, pp. 1944-1956, 1944, doi: 10.11591/ijece.v9i3.pp1944-1956.

[37] K. Anitha and S. Usha, "A simplified MAC protocol for energy efficiency in wireless sensor network," Journal of Advanced Research in Dynamical and Control Systems, vol.11, no. 3, pp. 1919-1927, 2019.

[38] K. Anitha and R. Shahabadkar, "EH-MAC: extensive hybrid medium access control mechanism for improving network lifetime and communication efficiency," International Journal of Innovative Technology and Exploring Engineering (IJITEE), vol. 9, no. 2, Dec. 2019.

\section{BIOGRAPHIES OF AUTHORS}
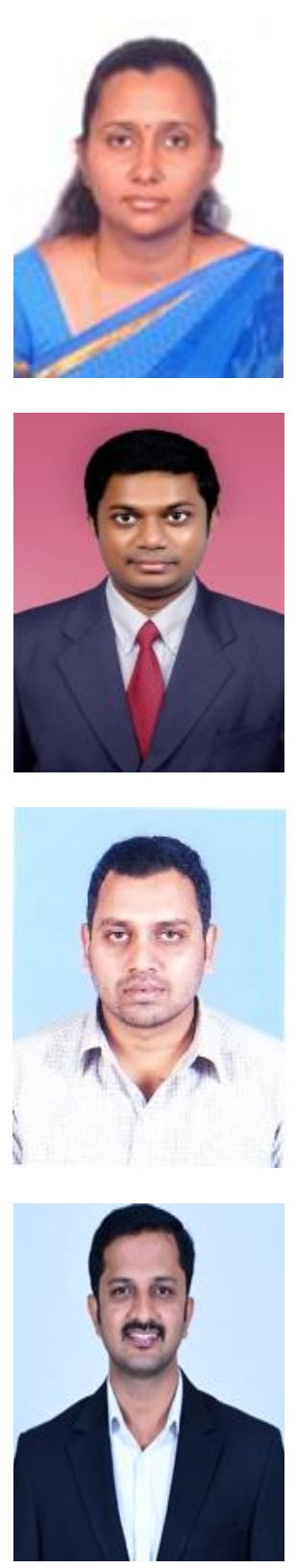

Anitha Krishna Gowda (D) SI SC P received her Ph.D. Degree in Computer Science and Engineering from Visvesvaraya Technological university, Belagavi. Her area of interest includes Wireless Sensor Networks, Artificial Intelligence, Datascience and Internet of Things. She is Presently working as an Associate Professor in the Department of Computer Science and Engineering, Global Academy of Technology, Bengaluru, Karanataka, India. She can be contacted at email: anitharesearchvtu@gmail.com.

Ananda Babu Jayachandra (D) SC P received his Ph.D. degree in Computer Science and Engineering from Visvesvaraya Technological university, Belagavi. He is expertise in Wireless Sensor Networks, Artificial Intelligence and Datascience. He is Presently working as an Associate Professor in the department of Information Science and Engineering, Malnad College of Engineering, Hassan, Karanataka, India. He can be contacted at email: abj@mcehassan.ac.in.

Raviprakash Madenur Lingaraju (iD $8 \mathrm{SC}$ P is currently working as Associate Professor in Department of Computer Science and Engineering, Kalpataru Institute of Technology, Tiptur. His area of research includes image processing, computer networks, artificial intelligence. He is a member of MISTE and CSI. He can be contacted at email: raviprakashml@kittiptur.ac.in.

Vinay Doddametikurke Rajkumar (D) SC P worked as a Assistant Professor in the Department Computer Science Engineering, Channabasaveshwara Institute of Technology, Gubbi. He obtained his BE Degree Computer Science Engineering from Visvesvaraya Technological University, Belgavi. He received M.Tech. Degree in Computer Science and Engineering at Visvesvaraya Technological University, Belgavi.He is pursuing $\mathrm{PhD}$ in Computer Science Engineering, Malnad College of engineering, Hassan. His area of interest is in the field of digital image processing, steganography, machine learning and neural network. He is a member of MISTE. He can be contacted at email: vinu.ise@ gmail.com. 